\title{
MVZ nur zur Hälfte in vertragsärztlicher Hand
}

— Die KBV hat zum Stichtag 31.3.2010 aktuelle Daten zur Verbreitung und Organisationsform medizinischer Versorgungszentren (MVZ) in Deutschland veröffentlicht. Demnach gibt es zum Stichtag 1503 MVZ mit 7526 dort tätigen Ärztinnen und Ärzten. 1320 davon sind Vertragsärzte, 6206 angestellte Ärzte.

Ein MVZ besteht durchschnittlich aus fünf Ärzten mit steigender Tendenz, insbesondere im Krankenhaus-MVZ. 732 (48,7\%) MVZ befinden sich in vertragsärztlicher Hand, 578 (38,5\%) in der Trägerschaft eines Krankenhauses, wobei der Anteil der Krankenhausträgerschaft deutlich zunimmt.

Die am häufigsten gewählten Rechtsformen sind die GmbH (842), die GbR (459) und die Partnerschaftsgesellschaft (30). Häufigste Fachgruppen in einem MVZ sind Allgemeinärzte (1164) und Internisten (701). Den geringsten Beteiligungsgrad haben Urologen (94). Die

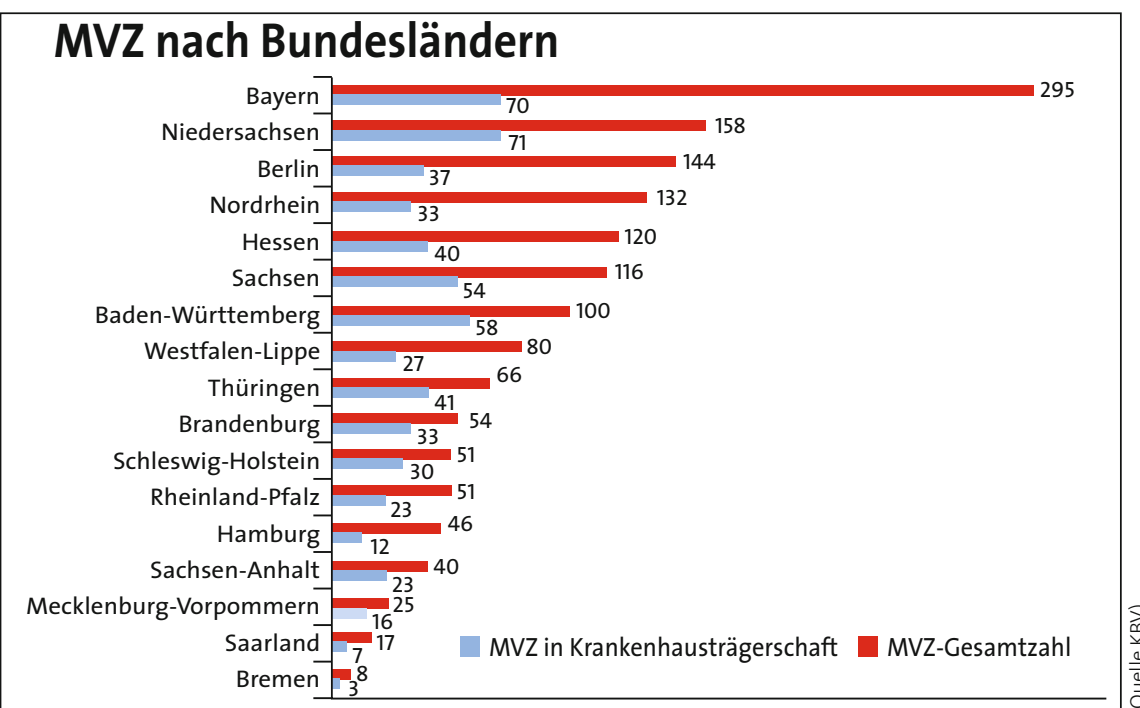

Die meisten MVZ gibt es in Bayern, die höchste Dichte aber in Berlin und in den neuen Bundesländern.

meisten MVZ gibt es in Bayern. Berlin (46), Thüringen (48), Sachsen (51) und Brandenburg (59) haben allerdings eine wesentlich höhere MVZ-Dichte je Arzt und damit den höchsten Organisationsgrad.

\section{MMW Kommentar}

Den höchsten Verbreitungsgrad an medizinischen Versorgungszentren haben somit die neuen Bundesländer. Das ist nicht überraschend, denn einerseits versucht man dort, durch eine solche Konzentration den Ärztemangel zu kompensieren. Andererseits kann die Entwicklung auch als Rückorientierung in die Zeiten der Polikliniken vor der Wende gewertet werden. Allerdings sind nur 46,3\% der MVZ in ländlichen Regionen angesiedelt, wo dies unter versorgungstechnischen Aspekten Sinn machen würde. 841 MVZ (56\%) befinden sich hingegen im städtischen Raum, wo eher betriebswirtschaftliche Überlegungen für die Gründung ausschlaggebend gewesen sein dürften.

Interessant ist dabei die zunehmende Beteiligungsform in Gestalt eines Angestelltenverhältnisses. Hier dürfte der Umstand eine führende Rolle spielen, dass mittler- weile weit über 50\% der Studienabgänger im Fach Humanmedizin weiblichen Geschlechts sind und auf diesem Weg versuchen, Beruf und Familie zu harmonisieren. Die Angestelltenkomponente ist in MVZ unter Krankenhausträgerschaft regelrecht dominant: 3039 Ärztinnen und Ärzte von 3083 sind in einem Krankenhaus-MVZ angestellt, während die Zahl der Angestellten in vertragsärztlich geführten MVZ stagniert. In den neuen Bundesländern überwiegt die Zahl der MVZ in Krankenhausträgerschaft deutlich. Überraschend ist dabei, dass auch im Krankenhaus-MVZ die Hausärzte mit 346 vor den Chirurgen mit 324 die Spitzenposition einnehmen. Die Bundesregierung will übrigens nun doch die ursprünglich angekündigte Eigentums- und Organisationsreform für MVZ nicht ändern. Während im Koalitionsvertrag noch festgelegt wurde, dass die Mehrheit der Geschäftsanteile und Stimmrechte in einem MVZ Ärztinnen und Ärzten zustehe, erklärt die Regierung nun ausdrücklich, sie halte an der Gründungsberechtigung von Krankenhäusern für MVZ fest. Dagegen prüft die Bundesregierung zurzeit die finanziellen und gesellschaftsrechtlichen Beziehungen der KBV zu den Gesellschaftern der Patiomed AG. Dabei soll geklärt werden, ob Patiomed die geplanten Ärztlichen Versorgungszentren als MVZ im Sinne des $\$ 95$ $S G B V$ gründen will. Zu den Gründungsgesellschaftern der umstrittenen Patiomed gehören die KVmed $\mathrm{GmbH}$ sowie die Deutsche Apotheker- und Ärztebank. Die $K V m e d ~ G m b H$ wiederum ist die Tochtergesellschaft der von der KBV 2008 ins Leben gerufenen Aeskulap-Stiftung. Auch die Asklepios Kliniken sind an der KVmed beteiligt. 